\title{
A better way to care for Long Term Care residents (LTC) in times of medical urgency: improving acute care for $\mathrm{LTC}$ residents.
}

\section{Leanna Wyer, Shawna Reid, Abraham Munene, Eddy Lang, Vivian Ewa, Heather Hair, Greta Cummings, Patrick McLane, Eldon Spackman, Peter Faris, Dominic Alaazi, Marian George, Jayna Holroyd-Leduc}

Background: Many LTC residents are transferred to Emergency Departments (EDs) with conditions that could be cared for in LTC, perhaps with additional support (e.g. Community Paramedics). Communication between sites and EDs has also been lacking. These lead to long lengths of stay in EDs, unnecessary use of resources, and sub-optimal health outcomes. Two INTERACT tools will support initial management of the concern at the LTC site. Then a Care and Referral Pathway will help facilitate needed conversations and optimal transfers between LTC and ED.

Implementation: Beginning in April 2019, standalone LTC sites in Calgary and Central zones have been invited to participate. Using a randomized stepped wedge design, we implement at 4-5 new sites every 3 months, with a total goal of implementing this change in 40 sites in Calgary and 9 sites in the Central zone. Early engagement with site medical directors, LTC and ED physicians, and managers at RAAPID (Referral, Access, Advice, Placement, Information and Destination) call centre and Community Paramedics was instrumental in getting the project initiated. Quarterly meetings with a project steering committee assists with ongoing project details and risk/issues. Operational leads and unit managers meet with our Senior Practice Consultant to be introduced to the project. This is followed by an implementation session at which site staff are given information about the specific tools and pathway, potential barriers are mitigated, and a site implementation plan is developed. Quarterly reports using data from a project Tableau dashboard are prepared by our Research Coordinator and distributed to LTC sites for them to monitor their performance compared to zone averages on a number of performance indicators.

Evaluation Methods: The project will be evaluated using both qualitative and quantitative measures. Key Performance Indicators include a reduction in transfers to EDs, improved satisfaction, and increased use of available resources. Residents, families and healthcare providers will participate in interviews or focus groups to assess their experiences with the interventions. Quantitative evaluation includes an economic analysis to determine how the interventions have led to cost savings within the healthcare system, as well as examination of the number transfers to ED, hospital admissions, calls to RAAPID, and visits by Community Paramedics. This will help to determine if the intervention has led to better resource utilization, increased satisfaction among residents and families, and improved patient and health system outcomes. At this stage of the project, no unintended consequences have been identified.

Results: Currently, we have implemented at 6 of 11 Cohorts (26 sites). Data from April 2019 (start of project) until December 2020 show a downward trend in number of ED visits and 
hospital admissions, as well as increased utilization of RAAPID. Formal evaluation will be completed when the project ends in June 2022. Given the COVID-19 pandemic, it is important to note that this may have an effect on our current trends and this will further be explored at the end of the project period. Anecdotal evidence is also beginning to indicate success of the right care being provided in the right place.

\section{Advice and Lessons Learned:}

1) Firstly, partnerships with key stakeholders are vital to ensure successful utilization of the LTC-ED Care and Referral pathway. Specifically, RAAPID is key to the facilitation of communication between LTC sites and the EDs, and the services provided by Community Paramedics allow many residents to remain at their LTC homes.

2) Secondly, good engagement with Site Medical Directors and Operational leads is needed to ensure LTC staff and physicians are supported to use the interventions, and to care for their residents on site if appropriate.

3) Finally, tailored implementation strategies for each individual LTC site (and units in some cases) help mitigate site specific barriers, leverage strengths, and work within the site culture. 